\title{
LOWER BOUNDS FOR CLASS NUMBERS OF REAL QUADRATIC AND BIQUADRATIC FIELDS
}

\author{
(Communicated by Larry J. Goldstein)
}

\section{R. A. MOLLIN}

\begin{abstract}
In this paper we provide lower bounds for class numbers of real quadratic fields of Richaud-Degert type, as well as for class numbers of related real biquadratic fields.
\end{abstract}

Introduction. Lower bounds for class numbers of real quadratic fields have been studied by $\mathrm{H}$. Hasse [6] and $\mathrm{H}$. Yokoi [15-16]. We generalized their results in [11] subject to a certain restriction which we are able to eliminate in this paper. Moreover, we use the techniques provided by the study of the quadratic field case to provide lower bounds for class numbers of biquadratic fields of type $Q\left(\sqrt{D^{2}+4 d}, \sqrt{D^{2}+\alpha d}\right)$ where $D>0, d$ divides $D, \alpha \in\{1,2\}$, and both $D^{2}+4 d>1$ and $D^{2}+\alpha d>1$ are square-free.

1. Lower bounds for class numbers of real quadratic fields. We begin with a definition. If $n$ is a positive square-free integer and $t$ is any positive integer, then we say that a rational integral solution $(u, v)$ of the diophantine equation $x^{2}-n y^{2}=$ $\pm 4 t$ is a trivial solution when $t=m^{2}$ and $m$ divides both $u$ and $v$. Otherwise $(u, v)$ is called a nontrivial solution. The following generalized Davenport-Ankeny-Hasse (D-A-H) lemma is proved in [12].

LEMMA 1.1. Let $n$ be a square-free positive integer and let $t$ be any positive integer. Suppose that $(T+U \sqrt{n}) / \sigma$ is the fundamental unit of $K=Q(\sqrt{n})$ where $\sigma=2$ if $n \equiv 1(\bmod 4), \sigma=1$ otherwise; and $T^{2}-n U^{2}=\sigma^{2} \delta$, (i.e., $\left.\delta= \pm 1\right)$. If there exists a nontrivial solution to the diophantine equation $x^{2}-n y^{2}= \pm \sigma^{2}$ then

$$
t \geqslant((2 T / \sigma)-\delta-1) / U^{2} .
$$

We use the above to prove the following generalization of [11, Theorem 1], wherein we were forced to assume odd class number, in view of the more restrictive (D-A-H) lemma stated therein. Before proceeding with the result some comments on notation are in order. In what follows $h(n)$ will denote the class number of $K=$ $Q(\sqrt{n})$, and $C_{K}$ will denote the class group of $K$. When referring to a prime $\mathscr{P}$ of the ring of integers of $K$ we will say that $\mathscr{P}$ is a $K$-prime.

Received by the editors May 7, 1986 and, in revised form, July 29, 1986.

1980 Mathematics Subject Classification (1985 Revision). Primary 12A50, 12A25: Secondary 12A95, $12 \mathrm{~A} 35$ 
THEOREM 1.1. Let $n, U, \sigma, \delta$ and $K$ be as in the statement of Lemma 1.1. If $p$ is a rational prime which splits in $K$ then

$$
h(n) \geqslant\left\{\log \left[\left(2\left(\sqrt{U^{2} n+\sigma^{2} \delta}\right) / \sigma\right)-\delta-1\right]-2 \log U\right\} / \log p .
$$

Proof. If $\mathscr{P}$ is a $K$-prime above $p$ and $k$ is the order of the class of $\mathscr{P}$ in $C_{K}$ then $k$ divides $h(n)$. Moreover, since $p$ splits in $K$ then $x^{2}-n y^{2}= \pm \sigma^{2} p^{k}$ has a nontrivial integral solution $(x, y)$. Thus we may invoke Lemma 1.1 to get

$p^{h(n)} \geqslant p^{k} \geqslant[(2 T / \sigma)-\delta-1] / U^{2}$.

However $T=\sqrt{U^{2} n+\sigma^{2} \delta}$ whence

$$
h(n) \geqslant\left\{\log \left[\left(\left(2 \sqrt{U^{2} n+\sigma^{2} \delta}\right) / \sigma\right)-\delta-1\right]-2 \log U\right\} / \log p .
$$

Q.E.D.

The following corollaries generalize and refine Mollin [10, Corollaries 3-6], which in turn generalized Hasse's [6, Satzen 2a-2c, p. 58]. The hypothesis and notation of Theorem 1.1 remain in force.

In the following we avoid the case $n=D^{2}-1$, as did Hasse in [6]. The reason is that $h(n)$ is known to be larger than 1 for all $D>2$ since such $n$ cannot be of the form $q, 2 p$, or $q_{1} q_{2}$ where $q$ is prime, $p \equiv 3(\bmod 4)$ is prime and $q_{1} \equiv q_{2} \equiv 3$ $(\bmod 4)$ are prime.

Corollary 1.1. Let $n=D^{2}+1$. If $p$ is a prime which splits in $K$ then

(a) If $r=1, D$ is even and $D>2 p$ then $h(n)>1$ and the following bound holds:

$$
h(n) \geqslant[\log (D / 2)] / \log p \text {. }
$$

(b) If $r=1, D$ is odd and $D>p / 2$ then $h(n)>1$ and the following bound holds:

$$
h(n) \geqslant[\log (2 D)] / \log p .
$$

Proof. From [3], the fundamental unit of $K$ is $D+\sqrt{n}$ (see also [4]).

(i) If $r=1$ and $D \neq 2$ is even then $T=2 D, U=\sigma=2$, and $\delta=-1$. Thus the inequality in (a) follows from Theorem 1.1. Furthermore, since $D>2 p$ then

$$
((2 T / \sigma)-\delta-1) / U^{2}=D / 2>p
$$

whence $h(n)>1$.

(ii) If $r=1$ and $D$ is odd then $T=D$ and $U=\sigma=1=-\delta$. Therefore the inequality in (b) follows from Theorem 1.1. Also since $D>p / 2$ then

$$
((2 T / \sigma)-\delta-1) / U^{2}=2 D>p
$$

whence $h(n)>1$. Q.E.D.

At this juncture it is worth commenting upon the strength of the bounds in Corollary 1.1. It is well known that there are infinitely many square-free integers of the form $n=D^{2}+1$. In point of fact Iwaniec [7] has shown that there are infinitely many such $n$ which are divisible by at most two primes. As noted in the discussion preceding Corollary $1.1, h(n)=1$ only if $n$ is divisible by at most two primes. However, Corollary 1.1 yields that all but finitely many of these remaining $n$ have $h(n)>1$. Let $p$ be an odd prime dividing $D$. Then by Corollary 1.1(b) we have that

$$
h(n) \geqslant \log (2 D) / \log p>1 \text {. }
$$


Therefore $h(n)>1$ for all $n=D^{2}+1$ with $D>1$ odd. If $D$ is even and $p$ is an odd prime dividing $D$ with $D>2 p$ then by Corollary 1.1 (a) $h(n)>1$. This latter case has further ramifications which deserve discussion. Chowla [2] conjectured that for primes of the form $D^{2}+1$ with $D>26$ we have $h(n)>1$. In Mollin [13] we proved that $h(n)>1$ for all square-free integers of the form $n=D^{2}+1$ where either $n$ is composite or $n$ is prime and $D \neq 2 p$ for any odd prime $p$. This is also the situation excluded by Corollary 1.1(a). Furthermore in [13] we proved that $h(n)=1$ is tantamount to several criteria among which is that the least prime quadratic residue modulo $n$ is in fact $p$, when $D=2 p$. It is a difficult problem to determine when such a least prime quadratic residue exists (e.g., see Chowla [1]). Another criterion for $h(n)=1$ which we displayed in [13] is that $-x^{2}+x+p^{2}$ is equal to a prime for all integers $x$ with $1<x<p$. Although the latter seems tractable on the surface, it too is difficult. However, in [14] Mollin and Williams were able to achieve the Chowla conjecture under the assumption of the generalized Riemann hypothesis. Now we turn back to further consequences of Theorem 1.1.

COROllaRy 1.2. Let $n=D^{2}+r$ where $|r|=4$. If $p$ is a prime which splits in $K$ then

(a) If $r=4$ and $D>p$ then $h(n)>1$ and the following bound holds:

$$
h(n) \geqslant[\log D] / \log p .
$$

(b) If $r=-4$ and $D>p+2$ then $h(n)>1$ and the following bound holds:

$$
h(n) \geqslant[\log (D-2)] / \log p .
$$

Proof. By [3] the fundamental unit of $K$ is $(D+\sqrt{n}) / 2$ (see also [5]). Thus $T=D, U=1, \sigma=2$, and $\delta=-r /|r|$. Hence the inequalities in (a) and (b) follow from Theorem 1.1. Moreover if $r=4$ and $D>p$ then

$$
((2 T / \sigma)-\delta-1) / U^{2}=D>p
$$

whence $h(n)>1$. Finally if $r=-4$ and $D>p+2$ then

$$
((2 T / \sigma)-\delta-1) / U^{2}=D-2>p,
$$

whence $h(n)>1$. Q.E.D.

COROLlaRY 1.3. Let $n=D^{2}+r, D>0$, where $|r| \neq 1$ or 4 and $r$ divides $4 D$. If $p$ is a prime which splits in $K,|r|>\sigma^{2} p$, and $r>-\sigma D$ if $r<0$ then $h(n)>1$ and the following bound holds:

$$
h(n) \geqslant\left\{\log \left[\left(2 D^{2}|r|+r|r|-r^{2}\right) / 2 \sigma^{2} D^{2}\right]\right\} / \log p .
$$

Proof. By [3] (see also [4-5]) the fundamental unit of $K$ is $\left(2 D^{2}+r+2 D \sqrt{n}\right) /|r|$. If $\sigma=1$ then $T=\left(2 D^{2}+r\right) /|r|, U=2 D /|r|$, and $\delta=1$. If $\sigma=2$ then $T=$ $\left(4 D^{2}+2 r\right) /|r|, U=4 D /|r|$, and $\delta=1$. The inequality now follows from Theorem 1.1. Furthermore if $\sigma=1,|r|>p$, and $r>-D$ if $r<0$ then

$$
((2 T / \sigma)-\delta-1) / U^{2}=\left\{\begin{array}{ll}
r & \text { if } r>0 \\
-r-(r / D)^{2} & \text { if } r<0
\end{array}\right\}>p,
$$


whence $h(n)>1$. Finally, if $\sigma=2,|r|>4 p$, and $r>-2 D$ if $r<0$ then

$$
((2 T / \sigma)-\delta-1) / U^{2}=\left\{\begin{array}{ll}
r / 4 & \text { if } r>0 \\
-(r / 4)-(r / 2 D)^{2} & \text { if } r<0
\end{array}\right\}>p,
$$

whence $h(n)>1$. Q.E.D.

In Corollaries 1.2-1.3 we see that the lower bounds on the class numbers are nontrivial under the given hypotheses. Moreover, since the regulators of these Richaud-Degert type quadratic fields are small, then the Brauer-Siegel Theorem gives us that $h(n) \rightarrow \infty$ as $n \rightarrow \infty$; i.e., $h(n)$ grows with the discriminant. This is of course not the case with arbitrary real quadratic fields, since small regulators are rare in general.

Finally, since the results of the next section on biquadratic fields are based upon the bounds obtained in this section, no discussion of those bounds will be necessary.

\section{Lower bounds for real biquadratic fields.}

Theorem 2.1. Suppose that $D>0$ and $d$ are integers such that $d$ divides $D$. Let $K=Q\left(\sqrt{n_{1}}, \sqrt{n_{2}}\right)$ with $n_{1}=D^{2}+4 d>1$ and $n_{2}=D^{2}+\alpha d$ where $\alpha \in\{1,2\}$ and 3 divides $\left(2 D^{2} / d\right)+5$ when $\alpha=1$. Furthermore set $n_{3}$ equal to the square-free part of $n_{1} n_{2}$, and let $p$ be a rational prime which splits in $K$. If $n_{1}$ and $n_{2}$ are square-free then

(a) If $|d| \neq 1,(D, d) \neq(7,-7)$ when $\alpha=1$, and $(D, d) \neq(5,-5)$ when $\alpha=2$, then the following bounds hold:

$$
\begin{array}{ll}
h(K) \geqslant\left[\log \left(\left(D^{2}+d\right) / 3 d\right)\right] / \log p & \text { if } d>0 \text { and } \alpha=1 . \\
h(K) \geqslant\left[\log \left(-\left(D^{2}+4 d\right) / 3 d\right)\right] / \log p & \text { if } d<0 \text { and } \alpha=1 . \\
h(K) \geqslant\left[\log \left(\left(2 D^{2}+4 d\right) / d\right)\right] / \log p & \text { if } d>0 \text { and } \alpha=2 . \\
h(K) \geqslant\left[\log \left(-\left(2 D^{2}+8 d\right) / d\right)\right] / \log p & \text { if } d<0 \text { and } \alpha=2 .
\end{array}
$$

(b) If $|d|=1$ and $(D, d) \neq(3,-1)$ when $\alpha=2$, then the following bounds hold:

$$
\begin{array}{ll}
h(K) \geqslant[\log D] / \log p & \text { if } d=1 \text { and } \alpha=2 . \\
h(K) \geqslant[\log (D-2)] / \log p & \text { if } d=-1 . \\
h(K) \geqslant\left[\log \left(\left(D^{2}+1\right) / 3\right)\right] / \log p & \text { if } \alpha=d=1 .
\end{array}
$$

Proof. Let $F_{i}=Q\left(\sqrt{n_{i}}\right)$ for $i \in\{1,2,3\}$. To prove (a) we first show that $K$ is ramified over $F_{3}$ at some $F_{3}$-prime. Suppose that, on the contrary, $K$ is unramified everywhere over $F_{3}$. Thus, by the multiplicativity of ramification indices in towers, we have that every prime which ramifies in $F_{j}$ for $j \in\{1,2\}$, must ramify in $F_{3}$. Since $n_{j}$ is square-free then $n_{j}$ divides $n_{3}$ whence $d$ divides $n_{3}$. Since $|d| \neq 1$ then $n_{3}$ is divisible by a prime divisor $q>2$ of $d$. However $d^{2}$ divides $n_{1} n_{2}$ whence g.c.d. $\left(n_{3}, d\right)=1=$ g.c.d. $\left(n_{3}, q\right)$, a contradiction. We have shown that $K$ is ramified over $F_{3}$ at some $F_{3}$-prime. Therefore we may invoke [8] to get that $h\left(n_{3}\right)$ divides $h(K)$ (see also [9 and 10]). 
Now we show that the inequalities in (a) hold. Since 3 divides $\left(2 D^{2} / d\right)+5$ and $(D, d) \neq(7,-7)$ when $\alpha=1$, then by [4, Proposition 5, p. 141], the fundamental unit of $F_{3}$ is

$$
\left(2 D^{2}+5 d+6|d| \sqrt{n_{3}}\right) / 3|d| \text { when } \alpha=1 \neq|d| .
$$

Since $(D, d) \neq(5,-5)$ when $\alpha=2$, then by [5, Proposition 4, p. 298], the fundamental unit of $F_{3}$ is $\left(D^{2}+3 d+|d| \sqrt{n_{3}}\right) /|d|$ when $\alpha=2$ and $|d| \neq 1$. Moreover from [4] and [5 (ibid)] $\delta=1$ if $(D, d) \neq(7,-7)$ when $\alpha=1$. Therefore if $\alpha=1 \neq|d|$ then $n_{3}=\left(D^{4}+5 D^{2} d+4 d^{2}\right) / 9 d^{2}, \delta=\sigma=1$, and $U=2$; and if $\alpha=2$ and $|d| \neq 1$ then $n_{3}=\left(D^{4}+6 D^{2} d+8 d^{2}\right) / d^{2}$ and $\delta=\sigma=U=1$. We now may invoke Theorem 1.1 to get (for $|d| \neq 1$ ):

$$
\begin{aligned}
h\left(n_{3}\right) \geqslant \begin{cases}\left\{\log \left[2 \sqrt{\left(4\left(D^{4}+5 D^{2} d+4 d^{2}\right) / 9 d^{2}\right)+1}-2\right]-\log 4\right\} / \log p \\
\left\{\log \left[2 \sqrt{\left(\left(D^{4}+6 D^{2} d+8 d^{2}\right) / d^{2}\right)+1}-2\right]\right\} / \log p & \text { if } \alpha=2\end{cases} \\
= \begin{cases}\left\{\log \left[\left(D^{2}+d\right) / 3 d\right]\right\} / \log p & \text { if } \alpha=1 \text { and } d>0, \\
\left\{\log \left[-\left(D^{2}+4 d\right) / 3 d\right]\right\} / \log p & \text { if } \alpha=1 \text { and } d<0, \\
\left\{\log \left[2\left(D^{2}+2 d\right) / d\right]\right\} / \log p & \text { if } \alpha=2 \text { and } d>0, \\
\left\{\log \left[-2\left(D^{2}+4 d\right) / d\right]\right\} / \log p & \text { if } \alpha=2 \text { and } d<0 .\end{cases}
\end{aligned}
$$

This completes (a) since $h(K) \geqslant h\left(n_{3}\right)$. Note that although $h\left(n_{i}\right)$ divides $h(K)$ for $i \in\{1,2\}$ as well, $h\left(n_{3}\right)$ provides the largest bound, which is a straightforward but tedious check.

To verify (b) we first prove that $K$ is ramified over $F_{1}$ at some $F_{1}$-prime. Suppose that $K$ is unramified over $F_{1}$; then, as in the proof of (a), $n_{2}$ divides $n_{1}$. Therefore there exists an integer $l>1$ such that $D^{2}+4=\left(D^{2}+\alpha\right) l$; i.e., $D^{2}(l-1)=$ $-\alpha(l-1)+4-\alpha$, whence $l-1$ divides $4-\alpha$. If $\alpha=1$ then $l=2$ or 4 , whence $D$ is even, contradicting that $n_{1}$ is square-free. If $\alpha=2$ then $l=2$ or 3 . $l=2$ implies $D$ is even and $l=3$ implies $D^{2}=-1$, both being contradictions. Hence $K$ is ramified over $F_{1}$ at some $F_{1}$-prime. Now we invoke [8] again to get that $h\left(n_{1}\right)$ divides $h(K)$.

By [3] (see also [4-5]), the fundamental unit of $F_{1}$ for $|d|=1$ and $(D, d) \neq(3,-1)$ is $\left(D+\sqrt{n_{1}}\right) / 2$; that of $F_{2}$ for $d=1$ is $D+\sqrt{n_{2}}$ if $\alpha=1$ (observe that $-d=1=\alpha$ is not possible since $n_{2}$ is square-free), and for $|d|=1$ it is $D^{2}+d+D \sqrt{n_{2}}$ if $\alpha=2$. Therefore if $|d|=1$ we invoke Corollary 1.2 to get

$$
h\left(n_{1}\right) \geqslant \begin{cases}\log D / \log p & \text { if } d=1, \\ {[\log (D-2)] / \log p} & \text { if } d=-1 .\end{cases}
$$

Since $h(K) \geqslant h\left(n_{1}\right)$ by the above, then we have verified the first two inequalities of (b). Note that $h\left(n_{2}\right)$ divides $h(K)$ as well but $h\left(n_{1}\right)$ provides a larger bound.

Now we turn to the final inequality. From the above we have $h(K) \geqslant h\left(n_{1}\right)$ if $\alpha=d=1$, but we can say more in this case. First we claim that $K$ is ramified over $F_{3}$ at some $F_{3}$-prime. (Note that this does not occur in general when $\alpha=2$; and as 
mentioned above $\alpha=1=-d$ is not possible.) If $K$ is unramified over $F_{3}$ then, as in the proof of (a), every prime which ramifies in $F_{1}$ or $F_{2}$ ramifies in $F_{3}$. However, the fact that 3 divides $2 D^{2}+5$ guarantees that 3 ramifies in both $F_{1}$ and $F_{2}$, hence not in $F_{3}$, a contradiction. Now since $K$ ramifies over $F_{3}$ we may again invoke [8] to get $h\left(n_{3}\right)$ divides $h(K)$.

By [4] the fundamental unit of $F_{3}$ with $n_{3}=\left(D^{4}+5 D^{2}+4\right) / 9$ is

$$
\left(2 D^{2}+5+6 \sqrt{n_{3}}\right) / 3 \text {. }
$$

Therefore from Theorem 1.1 we get

$$
\begin{aligned}
h\left(n_{3}\right) & \geqslant\left[\log \left(2 \sqrt{\left(\left(4 D^{4}+5 D^{2}+4\right) / 9\right)+1}-2\right)\right] / \log p \\
& =\left[\log \left(D^{2}+1\right) / 3\right] / \log p .
\end{aligned}
$$

Q.E.D.

ACKNOWLEDGMENT. The author wishes to thank the referee for suggestions.

NOTE ADDED IN PROOF. The authors of [14] have found (under the assumption of the generalized Riemann hypothesis) all real quadratic fields of Richaud-Degert type having class number 1 (July 1987).

\section{REFERENCES}

1. S. Chowla, J. Cowles and M. Cowles, The least prime quadratic residue and the class number, J. Number Theory 22 (1986), 1-3.

2. S. Chowla and J. Friedlander, Class numbers and quadratic residues, Glasgow Math. J. 17 (1976), $47-52$.

3. G. Degert, Uber die Bestimmung der Grundeinheit gewisser reell-quadratischer Zahlkörper, Abh. Math. Sem. Univ. Hamburg 22 (1958), 92-97.

4. G. Frei, Fundamental systems of units in number fields $Q\left(\sqrt{D^{2}+d}, \sqrt{D^{2}+4 d}\right)$ with $d \mid D$, Arch. Math. 36 (1981), 137-144.

5. _ Fundamental systems of units in biquadratic parametric number fields, J. Number Theory 15 (1982), 295-303.

6. H. Hasse, Uber mehrklassige aber eingeschlechtige reell-quadratische Zahlkörper, Elem. Math. 20 (1965), 49-59.

7. H. Iwaniec, Almost-primes represented by quadratic polynomials, Invent. Math. 47 (1978), 171-188.

8. K. Iwasawa, $A$ note on class numbers of algebraic number fields, Abh. Math. Sem. Univ. Hamburg 20 (1956), 257-258.

9. R. Mollin, Class numbers and a generalized Fermat theorem, J. Number Theory 16 (1983), 420-429.

10. , Diophantine equations and class numbers, J. Number Theory 24 (1986), 7-19.

11. __ Lower bounds for class numbers of real quadratic fields, Proc. Amer. Math. Soc. 96 (1986), $545-550$.

12. On the insolubility of a class of diophantine equations and the nontriviality of the class numbers of related real quadratic fields of Richaud-Degert type, Nagoya Math. J. 105 (1987), 39-47.

13. Necessary and sufficient conditions for the class number of a real quadratic field to be one and a conjecture of $S$. Chowla (to appear).

14. R. Mollin and H. Williams, A conjecture of $S$. Chowla via the generalized Riemann hypothesis, Proc. Amer. Math. Soc. (to appear).

15. H. Yokoi, On real quadratic fields continuing units with norm -1, Nagoya Math. J. 33 (1968), $139-152$.

16. $106-115$ , On the fundamental unit of real quadratic fields with norm 1, J. Number Theory 2 (1970),

Department of Mathematics and Statistics, University of Calgary, 2500 University Drive N. W., Cai.gary, Alberta, Canada T2N 1N4 